\title{
Racial Disparities in Adverse Events in Children with Type 1 Diabetes
}

\author{
Terri H Lipman ${ }^{1,2,3}$, Steven M Willi2,3, Kathryn M Murphy²,3, Colin P Hawkes ${ }^{2,3}$ \\ ${ }^{1}$ University of Pennsylvania School of Nursing; ${ }^{2}$ Perelman School of Medicine; \\ ${ }^{3}$ Division of Endocrinology and Diabetes, Children's Hospital of Philadelphia, Philadelphia, PA, USA
}

\section{BACKGROUND}

There are racial disparities in the incidence, treatment and outcomes of children with type 1 diabetes (T1D). Patient SES has been proposed as a factor to explain the disparities. The purpose of this study was to compare missed diabetes appointments, emergency department visits and hospital admissions in African American(AA) versus white children with T1D, using health insurance status as a surrogate for SES.

\section{METHODS}

White and AA children $<18 \mathrm{yrs}$, with T1D $>1 \mathrm{yr}$, attending a large diabetes center in the US were included in a record review. Prevalence rates for missed appointments, ED visits and hospital admissions were examined for each race, with data stratified by insurance status.

\section{RESULTS}

1792 children [922 (51\%) male; $315(17.5 \%) A A]$ with a mean $( \pm S D)$ age of 13.2 (3.6) years and T1D duration of 5.9 (3.7) years, were included. Among those with public insurance- a higher proportion were AA $(57 \% \vee 19 \%, p<0.001)$, more AA children missed diabetes visits $(78 \%$ vs $54 \% p<0.001)$ required ED visits $(16 \% \vee 10 \%, p<0.001)$ and were admitted to the hospital $(18 \%$ vs $8 \%, p<0.001)$. Similarly, in those with private insurance, more AA children missed diabetes visits $(60 \% \mathrm{v}$ $40 \%, p<0.001)$ required ED visits $(15 \% \vee 4 \%, p<0.001)$ and were admitted to the hospital $(11 \% \vee 4 \%, p=0.002)$.

\section{CONCLUSIONS}

Racial disparities in the adverse events of children with T1D persist, even for those with private insurance. The extent of these disparities must be fully investigated to determine the contributing factors, including the impact of racial bias on treatment recommendations. Interventions must be developed to address these disparities so that optimal diabetes care can be provided to children of all racial, ethnic and SES backgrounds.

Table 1: Demographics for all patients diagnosed with T1D for $\geq 1 \mathrm{yr}$

\begin{tabular}{|c|c|c|c|c|c|c|}
\hline & \multicolumn{3}{|c|}{ Government Insurance } & \multicolumn{3}{|c|}{ Commercial Insurance } \\
\hline & $\begin{array}{l}\text { NHB } \\
(n=165)\end{array}$ & $\begin{array}{l}\text { NHW } \\
(n=272)\end{array}$ & $\mathbf{p}$ & $\begin{array}{l}\text { NHB } \\
(n=137)\end{array}$ & $\begin{array}{l}\text { NHW } \\
(n=1248)\end{array}$ & $\mathbf{p}$ \\
\hline Age, years & $\begin{array}{l}14.1 \\
(3.7)\end{array}$ & $\begin{array}{l}14.3 \\
(4.3)\end{array}$ & 0.8 & $\begin{array}{l}15.3 \\
(4.1)\end{array}$ & $\begin{array}{l}15.1 \\
(4.2)\end{array}$ & 0.6 \\
\hline Male, $\mathrm{n}(\%)$ & \begin{tabular}{|l}
86 \\
$(52.1 \%)$
\end{tabular} & $\begin{array}{l}139 \\
(51.1 \%)\end{array}$ & $0.8^{\#}$ & $\begin{array}{l}50 \\
(36.5 \%)\end{array}$ & $\begin{array}{l}690 \\
(55.3 \%)\end{array}$ & $<0.001$ \\
\hline $\begin{array}{l}\text { Duration of } \\
\text { T1D, years }\end{array}$ & $\begin{array}{l}6.2 \\
(4)\end{array}$ & \begin{tabular}{|l|}
6.7 \\
$(4.2)$
\end{tabular} & 0.2 & $\begin{array}{l}7.2 \\
(4.4)\end{array}$ & $\begin{array}{l}7.1 \\
(4.3)\end{array}$ & 0.7 \\
\hline $\begin{array}{l}\text { Annualized } \\
\text { Hemoglobin } \\
\text { A1c. \%* }\end{array}$ & $\begin{array}{l}9.8 \\
(8.4,11.5)\end{array}$ & $\begin{array}{l}8.6 \\
(7.4,9.7)\end{array}$ & $<0.001$ & $\begin{array}{l}9.2 \\
(8,10.5)\end{array}$ & $\begin{array}{l}8 \\
(7.2,8.9)\end{array}$ & $<0.001$ \\
\hline $\begin{array}{l}\text { Last } \\
\text { Hemoglobin } \\
\text { A1c, \%* }\end{array}$ & $\begin{array}{l}9.7 \\
(8.3,11.9)\end{array}$ & $\begin{array}{l}8.5 \\
(7.4,9.8)\end{array}$ & $<0.001$ & $\begin{array}{l}9.1 \\
(8,10.3)\end{array}$ & $\begin{array}{l}8 \\
(7.3,8.9)\end{array}$ & $<0.001$ \\
\hline $\begin{array}{l}\text { CGM used, } \\
\mathrm{n}(\%)\end{array}$ & \begin{tabular}{|l}
29 \\
$(17.6 \%)$
\end{tabular} & \begin{tabular}{|l}
107 \\
$(39.3 \%)$
\end{tabular} & $<0.001$ & $\begin{array}{l}37 \\
(27 \%)\end{array}$ & $\begin{array}{l}695 \\
(55.7 \%)\end{array}$ & $<0.001$ \\
\hline $\begin{array}{l}\text { Insulin } \\
\text { pump used, } \\
\mathrm{n}(\%)\end{array}$ & \begin{tabular}{|l}
35 \\
$(21.2 \%)$
\end{tabular} & $\begin{array}{l}122 \\
(44.9 \%)\end{array}$ & $<0.001$ & $\begin{array}{l}51 \\
(37.2 \%)\end{array}$ & $\begin{array}{l}798 \\
(63.9 \%)\end{array}$ & $<0.001$ \\
\hline
\end{tabular}

Government Insurance

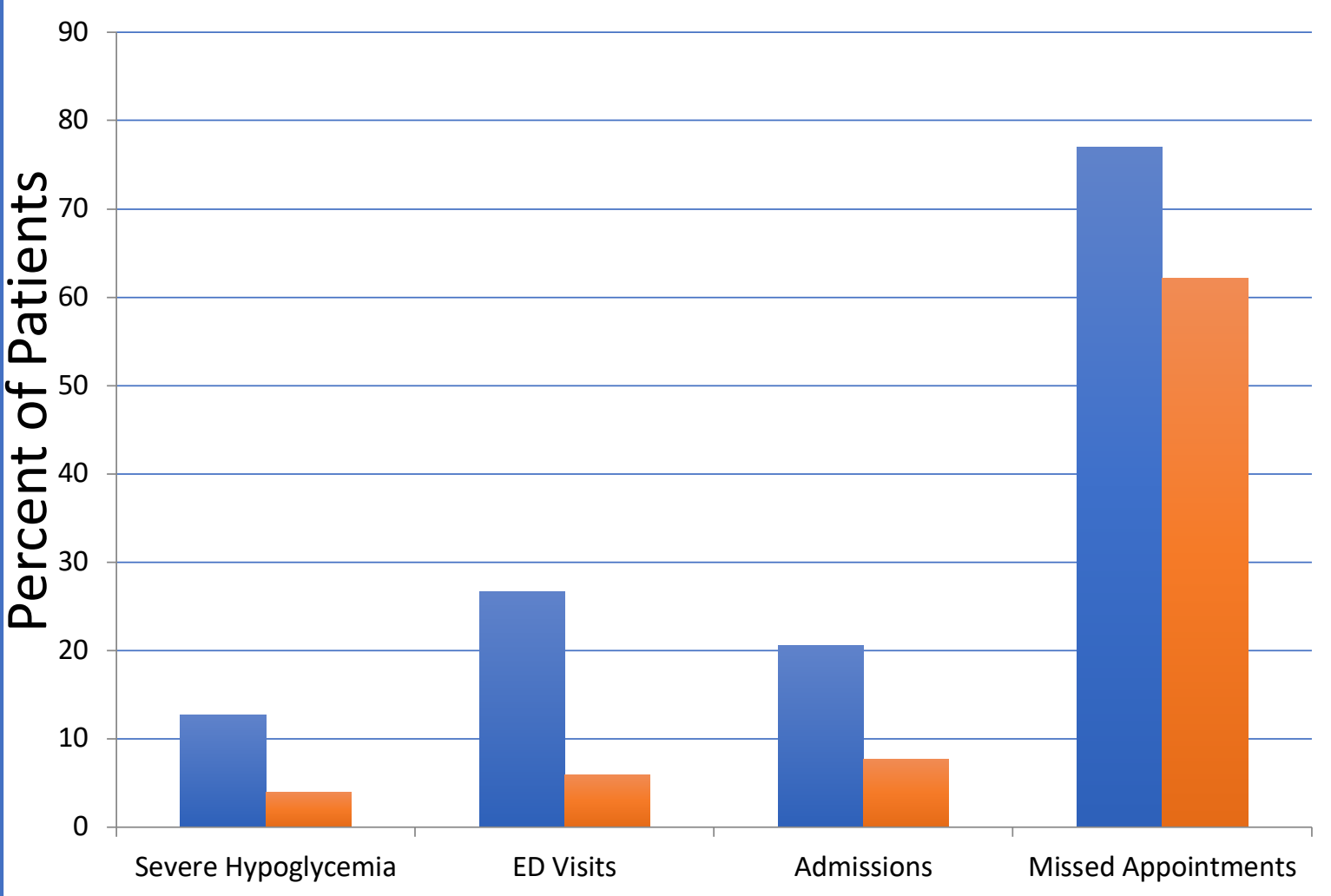

Commercial Insurance

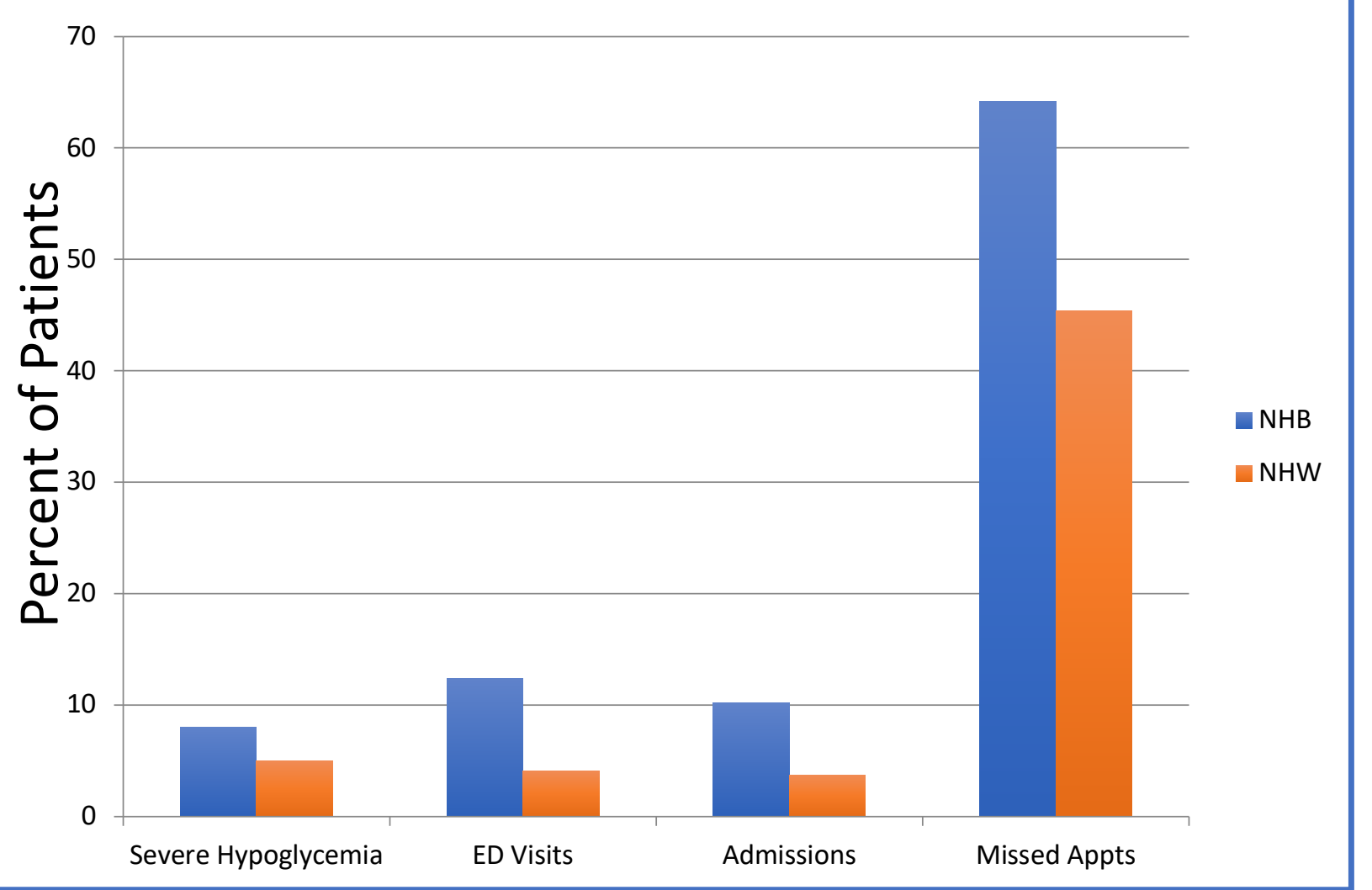

